\title{
Relevance of vancomycin susceptibility on patients outcome infected with Staphylococcus aureus
}

\author{
Ahmad Riyad Alsayed ${ }^{1}$, Malek Alzihlif², \\ Jamal Wadi Al Ramahi ${ }^{3}$
}

\section{Abstract}

Background: Staphylococcus aureus is a serious pathogen with high rates of complications. We aim to study the susceptibility and outcome of $S$. aureus infection.

Methods: A retrospective multicentre study conducted in three hospitals, Amman-Jordan. Between June 2013 and March 2014 laboratory records were reviewed for culture-positive samples growing $S$. aureus, also, medical records for the patients were reviewed for the demographic data, predisposing conditions, vancomycin MIC level, and outcome. Inpatients and outpatients were included, a case was classified as either hospital-associated (HA), community-associated (CA), or healthcare-associated (HCA). Data were entered as excel sheets and were statistically analyzed using SPSS version 21.

Results: A total of 127 patient (46\% MRSA) were culture-positive for $S$. aureus collected from different sources. Of these, eighty $(63 \%)$ were inpatients. High resistance rates to non- $\beta$-lactam antimicrobials were recorded. Glycopeptides agents were the antibiotics of choice for the treatment of infections caused by MRSA strains. Complications rates were higher in patients with MRSA infections including mortality, whereas hospital stay was longer for patients infected with MSSA.

Conclusion: Infection rates with MRSA were high among patients. There is a value for knowing vancomycin MICs for treatment of S. aureus and its implication for patients outcomes, though most outcomes were significantly worse due to MRSA infection.
1 Department of Clinical Pharmacy, School of Pharmacy, Applied Science Private University, Jordan.

2 Department of Pharmacology, School of Medicine, The University of Jordan, Amman, Jordan

3 School of Medicine, The University of Jordan, Amman, Jordan. Jordan Hospital Medical center, Amman, Jordan.

\section{Contact information:}

Jamal Wadi Al Ramahi MD, FIDSA.

Address: Adjunct, Assistant Professor of Medicine, School of Medicine, University of Jordan. Jordan Hospital Medical center. Amman, Jordan. 11118.

झjamalwadimd@yahoo.com 


\section{Introduction}

Staphylococcus aureus is a serious pathogen and a leading cause of both community- and healthcareassociated infections. Several factors may predispose patients to the development of

S. aureus infections such as intensive care units (ICUs) stay, long-term care facility residents, surgical patients, immunocompromised conditions, patients on hemodialysis, and various invasive procedures [1-2]. According to some healthcare systems, S. aureus was the second most common isolate, accounting for $20 \%$ of cases, and a prospective analysis in 49 hospitals in the united states between 1995-2002, reported that the proportion of MRSA increased from $22 \%$ in 1995 to $57 \%$ in 2001 [3]. Among patients consecutively admitted in 1993 to the adult intensive care unit (ICU) in Jordan University Hospital in Amman-Jordan, the most frequent species isolated was $S$. aureus [4]. A single center study conducted in Jordan hospital over a period of 3-year, found $S$. aureus the third most common blood isolate in the ICU after coagulase-negative staphylococci and E. coli, and accounting for 9.8 percent of cases [5]. Nonetheless, there has been in the mid-1990s an increase in the number of MRSA infections among patients who lacked proper health care exposure. This increase has been associated with the recognition of a different MRSA strains, such as community-associated MRSA (CA-MRSA), and these strains have rapidly spread in the world causing infections in the general population [6 -12]. In an analysis of 132 cases of MRSA bloodstream infections in patients admitted to a hospital in Atlanta- USA in 2004, molecular typing studies demonstrated that 34\% of isolates were CA-MRSA, which was geneticaIly distinct from the traditional strains HA-MRSA [13]. HA-MRSA strains carry a relatively large staphylococcal chromosomal cassette mec (SCCmec) belonging to type I, II, or III. These cassettes contain mecA gene, which is nearly universal, they are often resistant to many classes of antimicrobials.
Also, CA-MRSA isolates carry SCCmec type IV or $V$, though smaller elements and presumably mobile, but they are resistant to fewer non- $\beta$-lactam classes and frequently carry PVL genes [14]. Patients infected with MRSA strains need more care and have a poorer prognosis. Several studies have demonstrated increased mortality among patients infected with MRSA compared to MSSA infection [15-17].

This study aims to identify $S$. aureus susceptibility to vancomycin and the outcomes of its infection on patients in Jordan.

\section{Materials and Methods}

\section{Study Design and Settings}

This is an observational multicenter study included three private hospitals (Arab Medical Center, Al-Khalidi Hospital and Medical Center, and the University of Jordan hospital, Amman, Jordan. The study was approved by the institutional review board (IRB) in every hospital. Outpatients, and inpatients who had positive cultures of $S$. aureus were recruited over the period (June 2013 through March 2014). Records of the inpatients were reviewed, and patients were followed during hospitalization and after discharge. A case report form was filled included the following items: patients' demographic data; age, sex, weight, length of stay (LOS), admission location; ICU, medical, surgical, gynecology and pediatric. The chief complaint for admission, diagnoses, source of culture, infectious diagnosis, surgical procedures, previous antibiotic use, the antimicrobial susceptibility of $S$. aureus isolates, predisposing clinical conditions and comorbidities, malignancy, administration of steroids $>20 \mathrm{mg} /$ day of prednisone use or its equivalent for more than 14 days prior to specimen culture, antineoplastic chemotherapy in the 3 months prior to culture collection, longterm facility residence, indwelling catheters, intravenous drug use, diabetes mellitus, kidney disease, 
hemodialysis, skin or soft tissue lesions, respiratory illness, surgery wound and surgery requiring more than $48 \mathrm{~h}$ of hospitalization in the 30 days prior to admission, invasive procedures including cardiac catheterization, arterial angiogram, upper endoscopy, colonoscopy, bronchoscopy, tracheostomy, bone marrow aspiration, renal biopsy, hospitalization in the previous 12 months, and a history of previous MRSA infection and/or colonization. The patient's data about the use of antibiotics in the last three months and/or last week, frequent use of antibiotics prior vancomycin or other anti-MRSA agent exposure were recorded.

\section{Inclusion criteria}

S. aureus culture-positive specimens from inpatients and outpatients were included. The isolated $S$. aureus were classified as HA, CA, and HCA. CA-S. aureus is considered if the first isolate was recovered within $48 \mathrm{~h}$ of hospitalization, and if obtained from an outpatient source or the isolate was recovered within $48 \mathrm{~h}$ of hospitalization but believed to have had been incubating on admission, HCA cultures were considered from patients that frequently need healthcare attention or invasive procedures, but not admitted e.g. hemodialysis patients (CDC Definition). Non-duplicate strains for $S$. aureus isolates were considered. The outcome evaluated were vancomycin MIC distribution in relation to improved and discharged, discharged without improvement, switch to another antibiotic, relapse-progression, $\leq 30$-days readmission, infection-related readmission and, all cause of mortality among patients suffering of $S$. aureus infection.

\section{Identification of S. aureus}

S. aureus was identified by the routine standard hospital microbiology laboratory procedure, methicillin resistance was detected by the oxacillin and cefoxitin Kerby-Bauer disk diffusion, E-test (Retro C80TM, AB Biodisk, Sweden), or by VITEK 2 (bioMérieux) for identification and antibiotic suscep- tibility testing of gram-positive cocci was used to measure vancomycin MICs. Vancomycin susceptibility was defined according to The CLSI breakpoints (M100, Performance Standards for Antimicrobial Susceptibility Testing [18]); susceptible $\leq 2 \mathrm{mg} / \mathrm{L}$, intermediate $4-8 \mathrm{mg} / \mathrm{L}$, and concentrations $\geq 16 \mathrm{mg} /$ $\mathrm{mL}$ as resistant.

\section{Statistical Analysis}

Statistical analyses were performed using the SPSS 21 (Statistical Package for the Social Sciences, Version 21, Inc. IBM Corporation, Chicago, IL, USA), and tables were initially analyzed by Microsoft Excel (Microsoft Corporation). All data were calculated by non-parametric analysis due to low numbers or groups. Numbers were transformed to frequencies and analyzed as relative frequencies among MSSA and MRSA covariates and outcomes. Wilcoxon Sign Rank test was used to analyze MRSA and MSSA paired differences for the covariates and outcomes. Regression analysis for the relation between MIC and the days for the length of hospital stay. Kruskal Wallis tests to the asses the difference among means. P-value $<0.05$ was considered statistically significant.

\section{Results}

A total of 127 patients with their records for positive $S$. aureus cultures were recruited from the three hospitals in a 10-month period (June 2013-March 2014). Males constituted 87 (69\%) and females 40 (31\%). Out-patients were 47 (37\%); infected with $59.6 \%$ MSSA and $40.4 \%$ MRSA respectively, whereas in-patients were 80 (63\%); infected with 51.3\% MSSA and 49.7\% MRSA, respectively (Figure 1).

The age of the patients was distributed into groups, they were almost similar in numbers, but less for the age group $<20$, elder inpatients were more than the other age groups, and the outpatients elder age group were smaller in number. The total distribution of $S$. aureus susceptibility consis- 
ted of 69 (54.3\%) of MSSA and 58 (45.7\%) of MRSA isolates; inpatients were $51 \%$ and $49 \%$ for MSSA and MRSA respectively. The outpatients were $59.6 \%, 40.4 \%$, respectively. S. aureus did not grow from the abdomen, it grew from blood of inpatients. MSSA accounted for $75 \%$ of respiratory sample cultures which grew S. aureus from 49 patients (67.3\% MSSA) and (32.7\% MRSA), while skin grew 52 isolates, with MSSA $55.8 \%$ and MRSA $44.2 \%$ (Table 1).

Demographic features were collected from 80 patients with $S$. aureus infection based on their oxacillin susceptibility. Comorbidities; (BMl>25, CVD, diabetes, chronic kidney diseases, chronic respiratory diseases, and malignancy were significantly different in the distribution between MSSA and MRSA ( $P<$ 0.05). However, there was no significant difference for smoking, CNS disorders and skin diseases $(P>$ 0.05). There was a significant difference between the numbers of MSSA and MRSA for the use of antimicrobials either within the prior 3 months or 12 months of the study isolate $(P<0.05)$, and there were significant differences between both isolates based on the ward from which they were isolated $(P<0.05)$ (Table 2).

Both strains of $S$. aureus almost matched for their vancomycin susceptibility. The majority of MSSA isolates (20) and MRSA isolates (29) were in the range of $0.5-1.0 \mathrm{mg} / \mathrm{L}$. There were $4 \mathrm{MSSA}$ and one MRSA. 2 MSSA and 3 MRSA in the range MIC $>1-1.5 \mathrm{mg} / \mathrm{L}$, and 1 MSSA, 2 MRSA in the range $\mathrm{MIC}>1.5-2 \mathrm{mg} / \mathrm{L}$ and zero for both for the MIC >2 mg/L (Figure 2). Both strains were tested for susceptibilities based on the location of isolation as inpatients or outpatients. After the exclusion of the antimicrobials with zero susceptibility rates from MRSA like carbapenems and cephalosporines. In the MSSA there were statistically significant differences $(P<0.05)$ between inpatients and outpatients with better inpatient susceptibility for clindamycin and erythromycin, but better outpatient for quinolones, and TMP-SMX. For MRSA

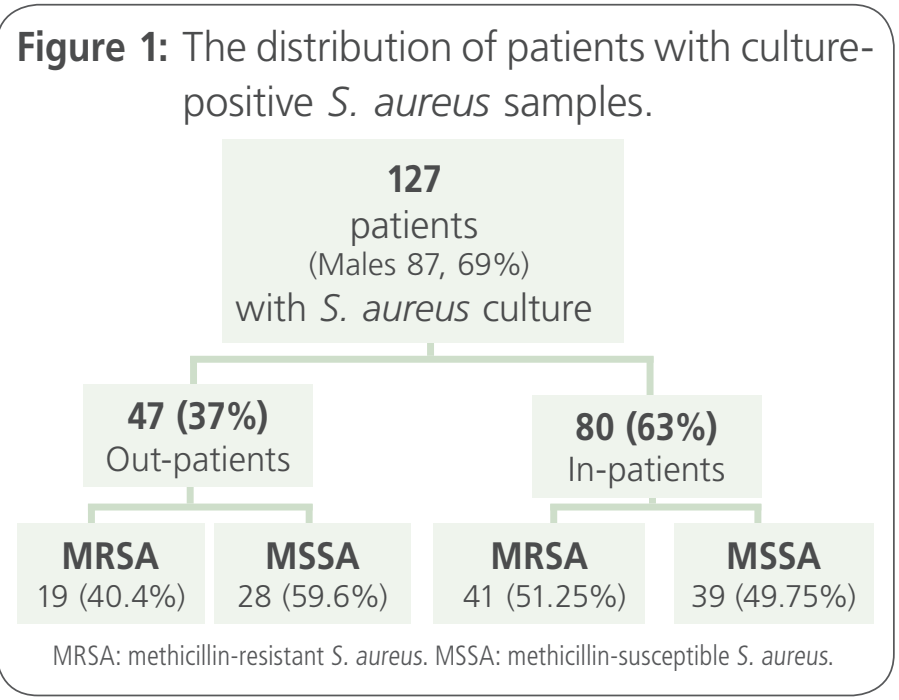

Table 1. Patients demographic features and characteristics.

\begin{tabular}{|c|c|c|c|c|c|c|c|}
\hline \multirow{2}{*}{$\begin{array}{c}\text { Demographic } \\
\text { data }\end{array}$} & \multicolumn{2}{|c|}{$\begin{array}{c}\text { All } \\
\text { Patients }\end{array}$} & \multicolumn{2}{|c|}{ Inpatients } & \multicolumn{2}{|c|}{ Outpatients } & \multirow[t]{2}{*}{$\mathbf{P}$} \\
\hline & $N$ & $\%$ & $\mathrm{~N}$ & $\%$ & $N$ & $\%$ & \\
\hline $\begin{array}{l}\text { Patients } \\
\text { Number }\end{array}$ & 127 & 100 & 80 & 63 & 47 & 37 & \\
\hline \multicolumn{8}{|l|}{ Gender } \\
\hline Male & 87 & 69 & 62 & 77.5 & 25 & 53.2 & \multirow{2}{*}{0.655} \\
\hline Female & 40 & 31 & 18 & 22.5 & 22 & 46.8 & \\
\hline \multicolumn{8}{|l|}{ Age (years) } \\
\hline$<20$ & 19 & 14.96 & 6 & 7.5 & 13 & 27.7 & \multirow[t]{4}{*}{0.144 . } \\
\hline $20-39$ & 38 & 32.13 & 24 & 30 & 14 & 29.7 & \\
\hline $40-59$ & 34 & 29.3 & 21 & 26.25 & 13 & 27.6 & \\
\hline$\geq 60$ & 36 & 23.78 & 29 & 36.25 & 7 & 15 & \\
\hline
\end{tabular}

Distribution of $S$. aureus by susceptibility

\begin{tabular}{|l|l|l|l|l|l|l|l|}
\hline MSSA & 69 & 54.3 & 41 & 51 & 28 & 59.6 & 0.18 \\
\hline MRSA & 58 & 45.7 & 39 & 49 & 19 & 40.4 & \\
\hline
\end{tabular}

\begin{tabular}{|c|c|c|c|c|c|c|c|}
\hline \multicolumn{3}{|c|}{$\begin{array}{l}\text { Distribution of } S \text {. aureus } \\
\text { by site }\end{array}$} & \multicolumn{2}{|c|}{ MSSAMRSA } & \multicolumn{2}{|c|}{ MSSAMRSA } & \\
\hline Abdomen & 0 & 0 & 0 & 0 & 0 & 0 & \\
\hline Blood & 22 & 8 & 21 & 7 & 1 & 1 & \\
\hline Bone & 4 & 3 & 3 & 2 & 1 & 1 & \\
\hline Respiratory & 33 & 16 & 17 & 14 & 1 & 2 & 0.423 \\
\hline Skin & 29 & 23 & 27 & 13 & 2 & 10 & \\
\hline Urine & 2 & 3 & 0 & 1 & 2 & 2 & \\
\hline Others & 9 & 4 & 2 & 0 & 7 & 4 & \\
\hline
\end{tabular}

P-values were calculated between the related groups i.e. MSSA versus MSSA and MRSA versus MRSA by Wilcoxon Sign Rank Test for inpatients and outpatients.

*P-value by the independent Kruskal Wallis Test. MSAA for the inpatients and outpatients and MRSA for the inpatients and outpatients were compared respectively. 
Table 2. Other demographic Features and characteristics based on $S$. aureus oxacillin susceptibility.

\begin{tabular}{|c|c|c|c|c|c|}
\hline \multirow[t]{2}{*}{ Demographic data } & \multicolumn{2}{|c|}{$\begin{array}{c}\text { All } \\
\text { Patients }\end{array}$} & \multicolumn{2}{|c|}{ Inpatients } & \multirow[t]{2}{*}{$\mathbf{P}$} \\
\hline & $\mathrm{N}$ & $\%$ & $\mathrm{~N}$ & $\%$ & \\
\hline $\begin{array}{l}\text { S. aureus infections } \\
(\mathrm{N}=80)\end{array}$ & 41 & 51 & 39 & 49 & \\
\hline \multicolumn{6}{|l|}{ Type of infection acquisition } \\
\hline CA & \multicolumn{2}{|c|}{6} & \multicolumn{2}{|r|}{2} & 0.014 \\
\hline $\mathrm{HCA}$ & \multicolumn{2}{|c|}{20} & \multicolumn{2}{|r|}{26} & 0.000 \\
\hline $\mathrm{HA}$ & \multicolumn{2}{|c|}{15} & \multicolumn{2}{|r|}{11} & 0.000 \\
\hline \multicolumn{6}{|l|}{ Type of infection acquisition } \\
\hline $\mathrm{BMI}>25$ & 14 & 34 & 21 & 83.9 & 0.000 \\
\hline Tobacco smoking & 16 & 39 & 16 & 41 & 1.00 \\
\hline CVD & 17 & 41.4 & 15 & 38.5 & 0.000 \\
\hline Diabetes mellitus & 18 & 44 & 20 & 51.3 & 0.000 \\
\hline Chronic kidney disease & 10 & 24.4 & 11 & 28.2 & 0.002 \\
\hline Chronic respiratory illness & 7 & 17 & 4 & 10.3 & 0.008 \\
\hline CNS disorders & 3 & 7.3 & 2 & 5 & 0.083 \\
\hline Malignancy & 9 & 21.9 & 2 & 5 & 0.003 \\
\hline Skin disease & 5 & 12.1 & 5 & 12.8 & 1.00 \\
\hline \multicolumn{6}{|l|}{ Previous hospital admissions } \\
\hline Within prior 3 months & 15 & 36.6 & 12 & 30.8 & \multirow{2}{*}{0.000} \\
\hline Within prior 12 months & 32 & 78 & 28 & 71.8 & \\
\hline $\begin{array}{l}\text { Antibiotics within the last } 3 \\
\text { months }\end{array}$ & 12 & 29 & 14 & 36 & 0.001 \\
\hline $\begin{array}{l}\text { Length of hospital stay in } \\
\text { days (range) }\end{array}$ & 14.4 & $1-81$ & 11 & $1-84$ & 0.000 \\
\hline \multicolumn{6}{|l|}{ Hospital Service } \\
\hline ICU & 9 & 21.9 & 12 & 30.7 & 0.003 \\
\hline Medical ward & 14 & 34.1 & 10 & 25.6 & 0.000 \\
\hline Surgical ward & 21 & 51.2 & 16 & 41 & 0.000 \\
\hline Pediatric ward & 4 & 10 & 2 & 5 & 0.046 \\
\hline All-cause mortality & 8 & 0.129 & 5 & 0.080 & 0.025 \\
\hline \multicolumn{6}{|c|}{$\begin{array}{r}\text { P value was tested by the Wilcoxon Sign Rank Test for the related } \\
\text { MSSA - MRSA paired differences. CA: community-associated, } \\
\text { HCA: healthcare-associated, HA: hospital-associated. CVD: } \\
\text { cardiovascular disease, CNS: central nervous system, ICU: } \\
\text { intensive care unit. }\end{array}$} \\
\hline
\end{tabular}

there was a significant differences $(P<0.05)$ between the inpatients and outpatients, better susceptibility rate in the outpatients for quinolones, gentamicin and rifampin, but better susceptibility rate among inpatients for erythromycin and TMPSMX (Figure 3).

S. aureus susceptibility to vancomycin, teicoplanin, tigecycline and rifampin was 100\%. Cephalosporines, carbapenems, penicillin and piperacillin/ tazobactam were $0 \%$ active against MRSA. After exclusion of the antimicrobial agents with both $100 \%$ susceptibility, and those with one agent with $0 \%$ susceptible for either strain from statistical analysis. Susceptibility differences for the other antimicrobial agents (quinolones, clindamycin, erythromycin, TMP/SMX and gentamicin) were significantly different in favor of MSSA $(P<0.001)$

\section{(Figure 4)}

The outcome rates for $S$. aureus were plotted as a strain specific relative frequency for MSSA and MRSA, the MICs relative frequency differences between both strains for the range $0.5-1.0 \mathrm{mg} / \mathrm{L}$ The other MIC ranges were not considered for analysis for being zero or few in numbers. For all outcomes the difference in the relative frequencies for patients with MSSA or MRSA was evident pointing at a worse MRSA outcome $(P=0.018)$, and for each outcome: improved and discharged $(P=0.008)$, relapse- 
Figure 3: The Rates of MSSA and MRSA susceptibilities for several antimicrobial agents in inpatients and outpatients. For MSSA, there were statistically significant differences $(P<0.05)$ between inpatients and outpatients for Clindamycin and for erythromycin, quinolones, and TMP-SMX. For MRSA there were significant differences $(P<0.05)$ between the inpatients and outpatients for quinolones, gentamicin and rifampin, and for erythromycin and TMP-SMX. By 2-tailed Wilcoxon Sign Rank Test.
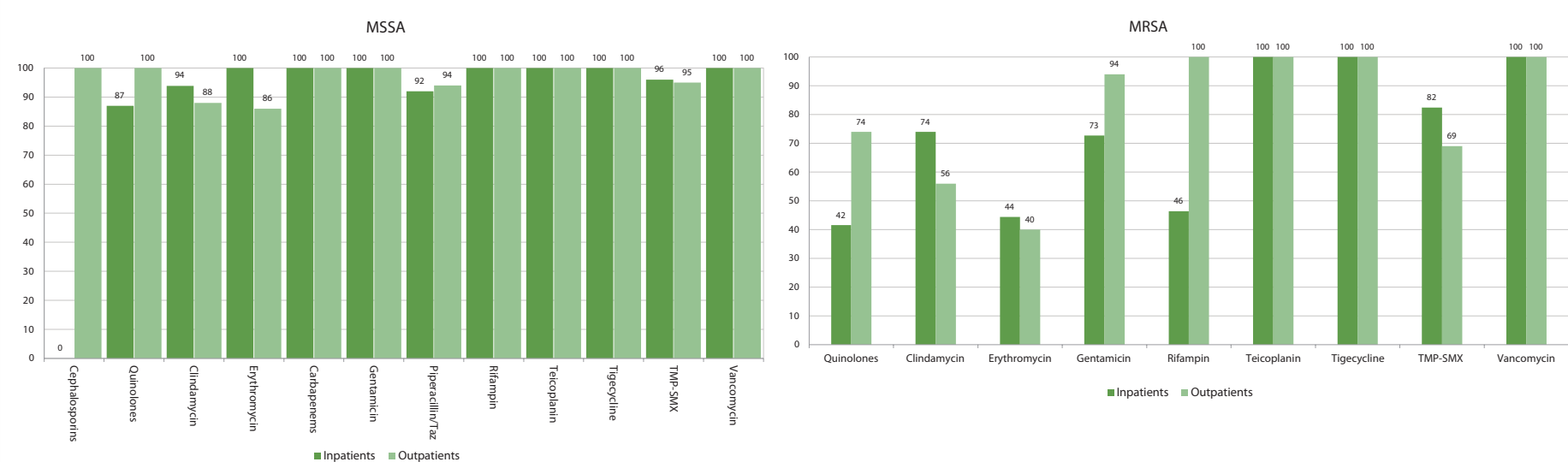

MRSA: methicillin-resistant Staphylococcus aureus. MSSA: methicillin-susceptible Staphylococcus aureus. Pip/Taz: piperacillin/Tazobactam. TMP-SMX: trimethoprim-sulfamethoxazole. Cephalosporins susceptibilities were not available for the inpatients and excluded from analysis.

Figure 4: Antimicrobials Susceptibility Rates for S. aureus isolates from the outpatients.

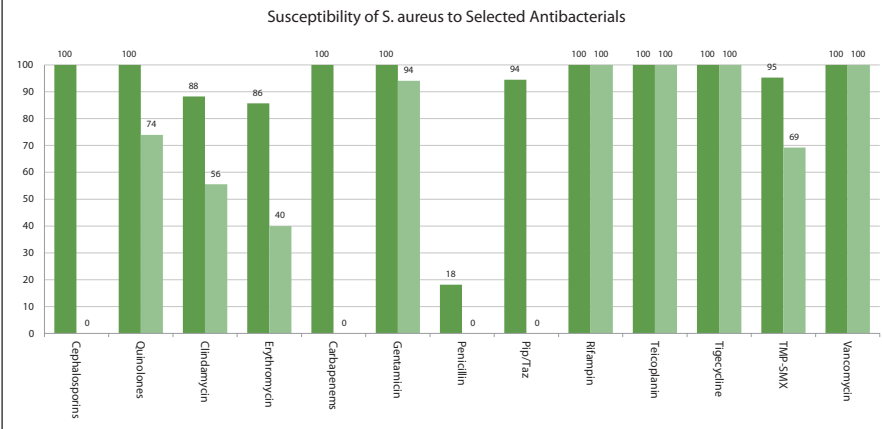

-MSSA \% $=$ MRSA\%

Pip/Taz: piperacillin/tazobactam. Quinolones: Ciprofloxacin, levofloxacin. Carbapenems: Imipenem, meropenem, ertapenem. Cephalosporins: Cefazolin, Cefuroxime, Ceftriaxone, Cefepime.

progression $(P=0.001), \leq 30$-days readmission $(P$ $=0.005)$, infection related readmission $(P=0.008)$, and all-cause mortality $(P=0.025)$ but was not significant for discharged without improvement $(P=$ $0.083)$ and switch to another antibiotic $(P=0.083)$,

\section{(Figure 5).}

Length of hospital stay was strongly associated with vancomycin susceptibility for MSSA, where it averaged 9.6 ( $n=4$, SD 5.86) when MIC was less than $0.5 \mathrm{mg} / \mathrm{L}, 14.06$ days ( $\mathrm{n}=20$, SD 20.79) for
Figure 5: The rates for patients with MSSA (20) and MRSA (29) for several measured outcomes. The Differences is measured by 2-taied Wilcoxon Sign Rank Test: improved and discharged ( $P=0.008)$, relapse-progression $(P=0.001), \leq 30$ days readmission $(P=0.005)$, infection related readmission ( $P=0.008)$, and all-cause mortality $(P=0.025)$ but was not significant for: discharged without improvement $(P=0.083)$ and switch to another antibiotic $(P=0.083)$.

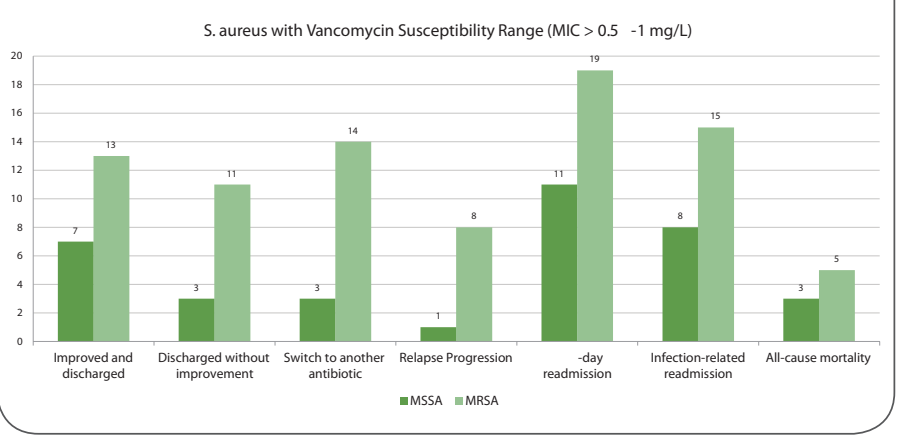


MIC > 0.5-1 mg/L and 45 days $(n=2$, SD 39.59) for MIC > 1-1.5 mg/L. Regression analysis showed perfect fit for the relation among MIC and length of hospital stay $\left(R=1, R^{2}=1\right)$, Kruskal Wallace test showed no significant differences among the three length of hospital stay for the cited number of patients for each groups $(P=0.386)$.

\section{Discussion}

Our study showed that the MRSA rates among all isolates of S. aureus were $45.7 \%$, these rates were lower than what was reported earlier by Frazee et al. (51\%) and Kaplan et al. (76.4\%) a decade earlier $[7,9]$ and $53.3 \%$ from Jordan [19]. The rates for the CA-MRSA (including HCA-MRSA) was 51.8\%, consistent with a previous study where it ranged 50.5$79.5 \%$ [12]. Patients with MRSA were more likely to have a risk factor. In this study, 78\% of MSSA cases and $71.8 \%$ of MRSA cases have a history of hospitalization in the last 12 months, those rates were not close to what was reported by Lescure, et al. which were 67\% MSSA and 85\% MRSA cases [20]. Comorbidities including BMI > 25, CVD, diabetes, chronic kidney diseases, chronic respiratory diseases, and malignancy were significantly higher in MRSA infected patients $(P<0.05)$. Smoking, CNS disorders and skin diseases were not different $(P>$ 0.05). There was a significant increase in the length of hospital stay and cost for patients with MSSA infection $(P<0.05)$, like a recent study by $E$. Y. Klein [21], possibly due to the relatively earlier and higher MRSA mortality rate in our patients $(P=0.025$ ). Other studies found higher mortality rate associated with MRSA infection and longer hospital length of stay $[16,17,22]$.

Our data showed that $S$. aureus was mostly concentrated in the susceptibility range $(0.5-1 \mathrm{mg} / \mathrm{L})$, with almost no distribution difference in the relative frequencies between MSSA and MRSA, and only fewer strains were in < MIC $0.5 \mathrm{mg} / \mathrm{L}$ and $>$ MIC $1 \mathrm{mg} / \mathrm{L}$. Though the standard of care in the treatment of MSSA are the penicillinase-resistant semisynthetic penicillins or cephalosporines. Nevertheless, studies have shown that elevated vancomycin MIC for MSSA strains was associated with more treatment failures and mortality [23, 24]. Eight patients (2 cancer patients) with MSSA infection were treated with vancomycin, 6/8 (75\%) had a poor outcome, and the other patients were younger (31 and 6 years old). Vancomycin is considered suboptimal therapy for the treatment of MSSA infection compared to the anti-staphylococcal $\beta$-lactams and is associated with the increased rates of treatment failure and high mortality rates. $[25,26]$ Despite the Infectious Diseases of America (IDSA) guidelines support using vancomycin for MRSA infections when are susceptible (MIC < $2 \mathrm{mg} / \mathrm{L})$, some studies and systematic reviews demonstrated treatment failure when patients were treated with vancomycin with MIC $\geq 1.5 \mathrm{mg} / \mathrm{L}$. This is due to the difficulty in attaining the PK/PD target of $A U C / M I C \geq 400$ with the clinical doses [27-33]. Furthermore, poorer prognosis was associated with vancomycin MIC level of $>1 \mathrm{mg} / \mathrm{dl}$ [34]. Noteworthy, in our patients both MRSA and MSSA susceptibility to vancomycin showed almost similar MIC distribution, lucky enough the vast majority fell $\leq 1 \mathrm{mg} / \mathrm{L}$, this would attain the PK/PD target when clinically achieving the serum trough levels of 15-20 mg/L. Also, our data demonstrated to some extent, that the higher $S$. aureus MIC for vancomycin, the longer the length of hospital stay for patients is noted, though regression and correlation among MICs and length of stay were perfect, but the difference between their means was not significant for the analyzed number of patients within each group ( $P=0.386)$.

For several antimicrobials, MSSA and MRSA were compared respectively as inpatients and outpatients. For the MSSA, clindamycin and erythromycin showed significantly better efficacy among inpatient against MSSA $(P<0.05)$, and in the outpatient quinolones and TMP-SMX were 
better $(P<0.05)$. For the MRSA, erythromycin and TMP-SMX showed significantly better anti-MRSA activity inpatient $(P<0.05)$, and in the outpatient, quinolones, gentamicin and rifampin were better $(P<0.05)$. The rest of the antimicrobials for both types of staphylococci showed no significant differences (Figure 3)

Susceptibility rates to the other antimicrobials were evaluated among outpatient MRSA isolates, these were $74 \%$ for quinolones and 69\% TMP/ SMX, respectively. An earlier study carried out in the Jordan University Hospital demonstrated that MRSA susceptibility to quinolones was $15 \%$, clindamycin $68 \%$, and gentamicin $68 \%$, respectively [35]. In the current study, a relatively higher susceptibility rates were observed for quinolones $74 \%$, clindamycin $56 \%$, and gentamycin 94\%, respectively. An older Saudi study from the 1980s [36] found imipenem to have had an excellent in vitro activity against MRSA isolates after vancomycin. Nevertheless, in this study the lack of sensitivity of MRSA isolates to $\beta$-lactam antibiotics exemplified by cephalosporines and carbapenems is consistent with other more recent studies [7, 37 38]. Our finding showed that the susceptibility to imipenem was 0\% among 35 tested MRSA isolates. The rates of in vitro ineffective $\beta$-lactam for MRSA cases were $100 \%$ in this study, while up to decade earlier it was reported to be ineffective up to $78.7 \%$ by using empirical $\beta$-lactam prescriptions, and carried a poor prognosis $[7,39]$. These results clearly demonstrated the escalating rates of resistance.

The outcomes of patients treatment were analyzed for the vancomycin (MIC range $0.5-1 \mathrm{mg} / \mathrm{L}$ ), they could not be analyzed for the other vancomycin MIC distributions as they were low in count. Treatment of patients infected with MRSA evidently pointed at worse outcomes $(P=0.018)$. The outcome of each patient when analyzed separately, was significant $(P$ $<0.05)$ for improved and discharged, relapse-progression, $\leq$ 30-days readmission, infection related readmission, and all-cause of mortality. However, the result was not significant $(P>0.05)$ for discharged without improvement or after the switch to another antibiotic.

\section{Conclusion}

The increasing MRSA rates leave limited treatment options. Evaluating

S. aureus susceptibility to vancomycin with a minimum inhibitory concentration may help in predicting outcomes, nonetheless, our study significantly demonstrated worse outcome with MRSA infection.

\section{Acknowledgement}

This manuscript is derived from a Master thesis project by Ahmad Al Syed PharmD, MSc, Ph.D. We thank the participating hospitals (Al Khalidi, Arab Medical center, andJUH) for their support during the conduct of the study.

\section{Disclosure}

All Authors declare no conflict of interest related to this article.

\section{References}

1. Wernitz MH, Swidsinski S, Weist K, Sohr D, Witte W, Franke $K P$, et al. Effectiveness of a hospital wide selective screening programme for methicillin-resistant Staphylococcus aureus (MRSA) carriers at hospital admission to prevent hospitalacquired MRSA infections. Clin Microbial Infect 2005; 11 (6):457-65

2. Huang SS, Yokoe DS, Hinrichsen VL, Spurchise LS, Datta R, Miroshnik I, Platt R. Impact of routine intensive care unit surveillance cultures and resultant barrier precautions on hospital-wide methicillin-resistant Staphylococcus aureus bacteremia. Clin Infect Dis 2006; 43 (8):971-8.

3. Wisplinghoff $H$, Bischoff $T$, Tallent SM, Seifert $H$, Wenzel RP, Edmond MB. Nosocomial bloodstream infections in US hospitals: analysis of 24,179 cases from a prospective nationwide surveillance study. Clin Infect Dis 2004; 39 (3):309-17.

4. Shehabi AA, Baadran L. Microbial infection and antibiotic resistance patterns among Jordanian intensive care patients EM H J 1996; 2 (3):515-520. 
5. Abu Ashoor JW Al-Qwasmeh W, Kamel M S. Prevalence and antimicrobial Susceptibility Patterns of Bloodstream Isolates at Jordan Hospital Intensive Care Unit: Three years Experience. JRMS 2011; 18 (3):80-86.

6. Dietrich DW, Auld DB, Mermel LA. Community-acquired methicillin-resistant Staphylococcus aureus in southern. New England children. Pediatrics 2004; 113 (4):e347-52.

7. Frazee BW, Lynn J, Charlebois ED, Lambert L, Lowery D, Perdreau-Remington F. High prevalence of methicillin-resistant Staphylococcus aureus in emergency department skin and soft tissue infections. Ann Emerg Med 2005; 1; 45 (3):311-20.

8. Fridkin SK, Hageman JC, Morrison M, Sanza LT, Como-Sabetti $\mathrm{K}$, Jernigan JA, et al. Methicillin-resistant Staphylococcus aureus disease in three communities. N Engl J Med 2005; 352 (14):143644.

9. Kaplan SL, Hulten KG, Gonzalez BE, Hammerman WA , Lamberth L, Versalovic J, et al. Three-year surveillance of communityacquired Staphylococcus aureus infections in children. Clin Infect Dis 2005; 40 (12):1785-91.

10. Rim JY, Bacon AE. Prevalence of community-acquired methicillinresistant Staphylococcus aureus colonization in a random sample of healthy individuals. Infect Cont Hosp Epidemiol 2007; 28 (9):1044-6.

11. Skov RL, Jensen KS. Community-associated meticillin-resistant Staphylococcus aureus as a cause of hospital-acquired infections. J Hosp Infect 2009; 73 (4):364-70.

12. McMullen KM, Warren DK, Woeltje KF. The changing susceptibilities of methicillin-resistant Staphylococcus aureus at a midwestern hospital: the emergence of "communityassociated" MRSA. Am J Infect Control 2009; 1; 37 (6):454-7.

13. Seybold U, Kourbatova EV, Johnson JG, Halvosa SJ, Wang YF, King MD, et al. Emergence of community-associated methicillinresistant Staphylococcus aureus USA300 genotype as a major cause of health care-associated blood stream infections. Clin Infect Dis 2006; 42 (5): 647-56.

14. Berglund C. Söderquist B. The origin of a methicillin-resistant Staphylococcus aureus isolate at a neonatal ward in Swedenpossible horizontal transfer of a staphylococcal cassette chromosome mec between methicillin-resistant Staphylococcus haemolyticus and Staphylococcus aureus. Clin Microbiol Infect 2008; 14 (11): 1048-1056.

15. Hamze M, Dabboussi F, Daher W, Izard D. Antibiotic resistance of Staphylococcus aureus at North Lebanon: place of the methicillin resistance and comparison of detection methods. Pathologie-biologie 2003; 51 (1):21-6.

16. Cosgrove SE, Sakoulas G, Perencevich EN, Schwaber MJ, Karchmer AW, Carmeli Y. Comparison of mortality associated with methicillin-resistant and methicillin-susceptible Staphylococcus aureus bacteremia: a meta-analysis. Clin Infect Dis 2003; 36 (1):53-9.
17. Shurland S, Zhan M, Bradham DD, Roghmann MC. Comparison of mortality risk associated with bacteremia due to methicillinresistant and methicillin-susceptible Staphylococcus aureus. Infect Cont Hosp Epidemiol 200; 28 (3):273-9.

18. CLSI. Clinical Laboratory and Standards Institute Method for dilution antimicrobial susceptibility tests for bacteria that grow aerobically. CLSI, 2013,Villanova, PA, USA.

19. Shehabi AA, AbuYousef R, Badran E, AlBakri AG, AbuQatouseh LF, Becker K. Major characteristics of Staphylococcus aureus colonizing J ordanian infants. Pediatr Int 2013; 55 (3):300-4.

20. Lescure FX, Locher G, Eveillard M, Biendo M, Van Agt S, Le Loup G, et al. Community-acquired infection with healthcareassociated methicillin-resistant Staphylococcus aureus: the role of home nursing care. Infect Cont Hosp Epidemiol 2006 ; (11):1213-8.

21. Klein YE, Jiang W, Mojica N, Tseng KK, McNeill R, Sara E. Cosgrove et al. National Costs Associated with MethicillinSusceptible and Methicillin-Resistant Staphylococcus aureus Hospitalizations in the United States, 2010-2014. Clin Infect Dis 2019; 68 (1):22-8

22. Graffunder EM, Venezia RA. Risk factors associated with nosocomial methicillin-resistant Staphylococcus aureus (MRSA) infection including previous use of antimicrobials. J Antimicrob Chemother 2002; 49 (6):999-1005..

23. Aguado JM, San-Juan R, Fernández-Ruiz M, Chaves F. Role of high vancomycin minimum inhibitory concentration in the outcome of methicillin-susceptible Staphylococcus aureus bacteremia. J Infect Dis 2012; 205 (6):1024-5.

24. Kalil AC, Van Schooneveld TC, Fey PD, Rupp ME. Association between vancomycin minimum inhibitory concentration and mortality among patients with Staphylococcus aureus bloodstream infections: a systematic review and meta-analysis. JAMA 2014; 312 (15):1552-64.

25. Lodise TP, McKinnon PS, Levine DP, Rybak MJ. Impact of empirical-therapy selection on outcomes of intravenous drug users with infective endocarditis caused by methicillin-susceptible Staphylococcus aureus. Antimicrob Agents Chemother 2007; 51 (10):3731-3.

26. Kim SH, Kim KH, Kim HB, Kim NJ, Kim EC, Oh MD, et al. Outcome of vancomycin treatment in patients with methicillinsusceptible Staphylococcus aureus bacteremia. Antimicrob Agents Chemother 2008; 52 (1):192-7.

27. Rybak MJ, Lomaestro BM, Rotscahfer JC, Moellering Jr RC, Craig WA., Billeter $M$, et al. Vancomycin therapeutic guidelines: a summary of consensus recommendations from the infectious diseases Society of America, the American Society of HealthSystem Pharmacists, and the Society of Infectious Diseases Pharmacists. Clin Infect Dis 2009; 49 (3):325-327. 
28. Hidayat .K, Hsu DI, Quist R, Shriner KA, Wong-Beringer A. Highdose vancomycin therapy for methicillin-resistant Staphylococcus aureus infections: efficacy and toxicity. Arch Intern Med 2006; 166:2138-2144.

29. Holmes NE, Turnidge JD, Munckhof WJ, Robinson JO, Korman TM. O'Sullivan MVN, et al. Antibiotic choice may not explain poorer outcomes in patients with Staphylococcus aureus bacteremia and high vancomycin minimum inhibitory concentrations. J Infect Dis 2011; 204:340-347.

30. Takesue Y, Nakajima K, Takahashi Y, Ichiki K, Ishihara M, Wada Y, et al. Clinical characteristics of vancomycin minimum inhibitory concentration of $2 \mathrm{mug} / \mathrm{ml}$ methicillin-resistant Staphylococcus aureus strains isolated from patients with bacteremia. J Infect Chemother 2011; 17:52-57.

31. Lodise TP, Miller CD, Graves J, Evans A, Graffunder E, Helmecke $\mathrm{M}$, et al. Predictors of high vancomycin MIC values among patients with methicillin-resistant Staphylococcus aureus bacteraemia. J Antimicrob Chemother 2008; 62:1138-1141.

32. Jacob JT, DiazGranados CA. High vancomycin minimum inhibitory concentration and clinical outcomes in adults with methicillin-resistant Staphylococcus aureus infections: a metaanalysis. Int J Infect Dis 2013; 17 (2):e93-e100.

33.Van Hal SJ, Lodise TP, Paterson DL. The clinical significance of vancomycin minimum inhibitory concentration in Staphylococcus aureus infections: a systematic review and meta-analysis. Clin Infect Dis 2012; 54 (6):755-71.
34. Kullar R, Davis SL, Levine DP, Rybak MJ. Impact of vancomycin exposure on outcomes in patients with methicillin-resistant Staphylococcus aureus bacteremia: support for consensus guidelines suggested targets. Clin Infect Dis 2011; 52 (8):97581.

35. Bazzoun D, Harastani H, Shehabi A, Tokajian S. Molecular typing of Staphylococcus aureus collected from a Major Hospital in Amman, Jordan. J Infect Dev Ctries 2014; 8 (4):441-447.

36. Ishag AJ, Durgham SM, Shibl AM. In vitro susceptibility of methicillin-resistant Staphylococcus aureus to imipenem and other antimicrobial agents. Chemioterapia: Chemioterapia. 1987 Aug; 6 (4):261-3.

37. Naimi TS, LeDell KH, Como-Sabetti K, Borchardt SM, Boxrud DJ. Etienne J, et al. Comparison of community- and health careassociated methicillin-resistant Staphylococcus aureus infection. JAMA 2003; 290:2976-2984.

38. Hankin A, Everett WW. Are antibiotics necessary after incision and drainage of a cutaneous abscess? Ann Emerg Med 20017; 50 (1), 49-51.

39. Soriano A, Marco F, Martínez JA, Pisos E, Almela M, Dimova VP, et al. Influence of vancomycin minimum inhibitoryconcentration on the treatment of methicillin-resistant Staphylococcus aureus bacteremia. Clin Infect Dis 2008; 46:193-200.

\section{Publish in The International Arabic Journal of Antimicrobial Agents}

The Journal is an open access peer-reviewed journal that publishes scientific papers about all aspects of antimicrobials. The journal will publish original research articles, reviews, brief reports and case reports dealing with basic and clinical antibacterial agents, antiviral, antiprotozoals, antituberculuous, antifungal and antihelminthes agents. All manuscripts must be prepared in English, and are subject to a rigorous and fair peer-review process. Accepted papers will immediately appear online. The journal aims to advance the knowledge, attitude and the research of chemotherapy in the Arabic world in cooperation with international, national scientific and public societies as well as research centers with similar aims and objectives. 